\title{
Advanced neural network systems for solving complex real problems
}

\section{Special Issue of IWANN 2019}

\author{
Olga Valenzuela ${ }^{1}$ - Fernando Rojas ${ }^{2}$ - Ignacio Rojas ${ }^{2}$
}

Published online: 1 June 2021

(c) The Author(s), under exclusive licence to Springer Science+Business Media, LLC, part of Springer Nature 2021

\section{Preface}

The 15th International Work-Conference on Artificial Neural Networks (IWANN) is a biennial meeting seeks to provide a discussion forum for scientists, engineers, educators and students about the latest discoveries and realizations in the foundations, theory, models and applications of systems inspired on nature, using computational intelligence methodologies, as well as in emerging areas related to the above items. As in previous editions, we strongly emphasize the wide range of topics comprised under the umbrella of IWANN2019 and, in particular, we focus on trending topics such as Deep Learning and Big Data.

This approach will be both theoretical and practical, through invited talks, tutorials, workshops, presentation of demos and functional prototypes. Besides, we offer the possibility to carry out a limited number of virtual presentations for those who want to participate but they have serious difficulties on travelling.

Since the first edition in Granada (LNCS 540, 1991), the conference has evolved and matured, and most of the topics involved have achieved a maturity and reinforced consolidation. The fifteenth edition of the IWANN conference "International Work-Conference on Artificial Neural Networks" was held in Gran Canaria, (Spain) during June 12-14, 2019. The list of topics in the successive Call for Papers has also evolved, resulting in the following list for the present edition:

1. Mathematical and theoretical methods in computational intelligence. Mathematics for neural networks. RBF structures. Self-organizing networks and methods. Support vector machines and kernel methods. Fuzzy logic. Evolutionary and genetic algorithms.

\section{Ignacio Rojas}

irojas@ugr.es

Olga Valenzuela

olgavc@ugr.es

Fernando Rojas

frojas@ugr.es

1 Department of Applied Mathematics, University of Granada, 18071 Granada, Spain

2 Department of Computer Architecture and Computer Technology, Information and Communications Technology Center (CITIC-UGR), University of Granada, 18071 Granada, Spain 
2. Neurocomputational formulations. Single-neuron modelling. Perceptual modelling. System-level neural modelling. Spiking neurons. Models of biological learning.

3. Learning and adaptation. Adaptive systems. Imitation learning. Reconfigurable systems. Supervised, non-supervised, reinforcement and statistical algorithms.

4. Emulation of cognitive functions. Decision Making. Multi-agent systems. Sensor mesh. Natural language. Pattern recognition. Perceptual and motor functions (visual, auditory, tactile, virtual reality, etc.). Robotics. Planning motor control.

5. Bio-inspired systems and neuro-engineering. Embedded intelligent systems. Evolvable computing. Evolving hardware. Microelectronics for neural, fuzzy and bioinspired systems. Neural prostheses. Retinomorphic systems. Brain-computer interfaces (BCI) Nanosystems. Nanocognitive systems.

6. Advanced topics in computational intelligence. Intelligent networks. Knowledgeintensive problem solving techniques. Multi-sensor data fusion using computational intelligence. Search and meta-heuristics. Soft Computing. Neuro-fuzzy systems. Neuroevolutionary systems. Neuro-swarm. Hybridization with novel computing paradigms.

7. Applications. Expert Systems. Image and Signal Processing. Ambient intelligence. Biomimetic applications. System identification, process control, and manufacturing. Computational Biology and Bioinformatics. Parallel and Distributed Computing. Human Computer Interaction, Internet Modeling, Communication and Networking. Intelligent Systems in Education. Human-Robot Interaction. Multi-Agent Systems. Time series analysis and prediction. Data mining and knowledge discovery.

In this edition of IWANN-2019, special workshop has been presented with the title: "Artificial Intellegence in nanophotonics", organized by Prof. Nikolay Zheludev and Prof. Cesare Soci. The Workshop on Artificial Intelligence in Nanophotonics aims to bring together the emerging community of researchers interested in:

1. The proliferation of AI approaches into the design of nanodevices, optimization of functionalities of artificial photonic nanostructures and material

2. Cognitive and neural functionalities in nanophotonic applications

A hands-on tutorial on Transfer Learning for Deep Neural Networks was carried out during IWANN 2019. The tutorial included a practical session with connection to the Barcelona Supercomputing Center (BSC), and was organized by D. Dario Garcia-Gasulla and D. Armand Vilalta.

The use of deep learning (DL) is one of the most active topics currently in the field of artificial neural networks, however training deep neural networks from scratch is not easy. Finding a good model for a given problem requires of huge amounts of data, lots of computational power, and a team of DL experts dedicated to the task for weeks. Since we cannot dedicate these resources for every single problem that may be appropriate for deep learning, the community has been actively looking for easier and faster solutions, mostly focused on the reuse of pre-trained deep learning models. This is the main goal of the transfer learning field, which seeks to exploit models designed and trained for a problem A to solve a potentially unrelated problem B. In edition of IWANN-2019, this tutorial introduced the two main approaches to transfer learning, fine tuning and feature extraction, detailing the benefits and handicaps of each one. The tutorial provided hands-on experience on running both types of transfer learning, while working on the CTE-POWER9 cluster hosted at BSC, which includes state-of-the-art Volta GPU racks.

At the end of the submission process of IWANN 2019, and after a careful peer review and evaluation process (each submission was reviewed by at least 2, and on the average 3.1 , program committee members or additional reviewers), 150 papers were accepted for 
oral or poster presentation, according to the recommendations of reviewers and the authors' preferences.

High-quality candidate papers (10 contributions) were invited to submit an extended version of their conference paper to be considered for special publication in this issue of Neural Processing Letters related with advanced neural network systems for solving complex real problems. These authors were selected after the recommendation of the reviewers of the conference papers, the opinion of the chairs of the different sessions and the guest editors. At least three independent and anonymous experts again carefully reviewed the extended versions and finally 7 papers were selected as appropriate for publication. In the present issue of Neural Processing Letters, it is a pleasure to present you these contributions that provide a clear overview of the thematic areas covered by the IWANN conference, ranging from theoretical aspects to real-world applications of nature-inspired system.

The first paper, "Integrating Machine Learning Techniques in Semantic Fake News Detection" by Adrian M.P. Brasoveanu et al., is focused in a hybrid model that combines machine learning, semantics and natural language processing in order to solve the problem of fake news detection. Fake news detection is a difficult problem due to the nuances of language. Understanding the reasoning behind certain fake items implies inferring a lot of details about the various actors involved. The authors present a hybrid solution to resolve this problem, combining machine learning, semantics and natural language processing. In this paper, the authors introduce a new semantic fake news detection method built around relational features like sentiment, entities or facts extracted directly from text. In the experiments result, it is shown that by adding semantic features the accuracy of fake news classification improves significantly.

In the paper entitled "A convolutional neural network framework for accurate skin cancer detection", by Karl Thurnhofer-Hemsi et al., the main goal is to presents a skin cancer detection method based on convolutional neural network $(\mathrm{CNN})$. The accurate prediction of the type of melanoma (heterogeneity and database size are the most important difficulties to overcome) is important to dispense the adequate treatment to the patient. Raw images are fed into a CNN to create both a plain and a hierarchical (with 2 levels) classifiers that are capable to distinguish between seven types of moles. The hierarchical system first determine if the patient is affected by malign cells and then specify the type of melanoma. This twolevel $\mathrm{CNN}$ is developed to deal with the unbalanced dataset because more than $50 \%$ of the images belong to the nevi class. Several convolutional neural network framework for accurate skin cancer detection were used due to their well-known effectiveness. Moreover, data augmentation was used to increase the number of input images. The HAM10000 dataset has been utilized for the experimental results and analysis of the proposed approaches. The dataset consists of 10015 dermatoscopic images, including a representative collection of all important diagnostic categories in the realm of pigmented lesions: Actinic keratoses and intraepithelial carcinoma / Bowen's disease (akiec), basal cell carcinoma (bcc), benign keratosis-like lesions (bkl), dermatofibroma (df), melanoma (mel), melanocytic nevi (nv, the class that has a greater number of images) and vascular lesions (vasc). The hierarchical classifier provides an effective way to discriminate between nevi and non-nevi moles with $96 \%$ accuracy.

Sebastien Delecraz et al., in the contribution entitled "Multimodal Machine Learning for Natural Language Processing: Disambiguating Prepositional Phrase Attachments with Images" presents an novel contribution focused on the problem of prepositional phrase (PP) attachment resolution (determining which word of a sentence is modified by a prepositional phrase), which has attracted the attention of many researchers in the field of Natural Language Processing (NLP). The paper present a strategy pipeline using visual information to 
disambiguate PP-attachments in sentences that describe a given image, showing a method for pairing objects detected in an image with noun phrases in its corresponding caption, and then selecting the best PP-attachment based on visual information. The authors have used the F30kE corpus enriched with 29,068 occurrences of prepositions manually attached to their governor, for the experiment section. The presented method yields improvements over a textonly syntactic parser, mainly for the subset of prepositions that encode location, leading to an increase of up to 17 points of attachment accuracy.

The paper entitled "Combining embeddings of input data for text classification", by Zuzanna Parcheta et al., presents a multi-input CNN which combines embeddings at the word, byte pair encoding and character levels, for text classification. The authors included experiments using four different text encoding techniques such as the Keras embedding layer, GloVe, BERT embeddings, and ParagraphVector. Experiments were conducted on seven datasets from different language families: English, German, Swedish and Czech. The authors have experimentally presented that in $78 \%$ of the cases the combination of different text encoding techniques improves the score obtained over using only one text encoding technique. By comparing different architectures, the paper has shown that the CNN architecture is the most suitable system for this problem.

The paper, presented by Cristiano Russo et al., "Knowledge Acquisition and Design Using Semantics and Perception. A Case Study for Autonomous Robots" proposes an extensible approaches for knowledge construction combining both ontologies, which are used for a standardized representation of knowledge in an automatic way, and machine learning techniques which helps in the object recognition task (CNN-based object classification).The first approach is an extension of a previous approach based on the definition of a multilevel semantic classiffier. The experiments carried out with novel features have shown improvement with respect to the previous implementation. Then, the authors have provided a use case in a real indoor environment using a humanoid robotic platform.

Oresti Banos et al., in the contribution entitled "Opportunistic activity recognition in IoT sensor ecosystems via multimodal transfer learning" presents a methodology for automatic recognition of physical activities. This typically involves various signal processing and machine learning steps used to transform raw sensor data into activity labels. The training of recognition systems continues to be both time and resource-consuming, as data sets must be collected ad-hoc for each specific sensor setup a person may encounter in their daily life. The authors present a new approach based on transfer learning to opportunistically train new unseen or target sensor systems from existing or source sensor systems. Two transfer models have been presented to translate recognition systems based on either activity templates or activity models, analyzing the behavior in a human-computer interaction scenario, where the transfer is performed in between wearable sensors placed at different body locations, and in between wearable sensors and an ambient depth camera sensor, presenting accuracy results. The proposed methodology supports the translation of activity recognition capabilities between sensor modalities without user or system designer's intervention.

Finally, the last selected paper entitled: "Cross-domain Polarity Models to evaluate User eXperience in E-learning" by Rosario Sanchis-Font et al., presents an approach that investigate how to automatically evaluate user experience in virtual learning environments, using Sentiment Analysis techniques. For this aim, a corpus with the opinions given by a total of 583 users (107 English speakers and 476 Spanish speakers) about three Learning Management Systems in different courses has been built. Authors apply machine learning tools to user's comments to obtain their polarity (positive, neutral or negative) using cross-domain models for both English and Spanish. Cross-domain models based on Deep Neural Networks (Convolutional Neural Networks, Transformer Encoders and Attentional BLSTM models) 
have been tested, and also commercial systems for the same task (MeaningCloud, Microsoft Text Analytics and Google Cloud). The experimental results were satisfactory, showing the usefulness of applying sentiment analysis tools on User eXperience evaluation.

The Guest Editors would like to express their gratitude to all the people who supported them in the compilation of this special issue, and specially to the contributing authors for their submissions and to the anonymous reviewers for their comments and useful suggestions in order to improve the quality of the papers. They would also like to express their gratitude to Editors-in-Chief M. Verleysen and M.H. Hassoun, for providing us with the opportunity to publish this set of selected papers in the present issue. It is a pleasure for us to invite all authors and interested readers of this issue to future IWANN conferences, which will be announced at http://iwann.ugr.es.

Publisher's Note Springer Nature remains neutral with regard to jurisdictional claims in published maps and institutional affiliations. 